\title{
The phthalocyanines applications in photodynamic therapy investigated by time-resolved and steady-state photothermal methods
}

\author{
Danuta Frackowiak, ${ }^{1}$ Krzysztof Wiktorowicz, ${ }^{2}$ Alfons Planner, ${ }^{1}$ \\ Aneta Waszkowiak, ${ }^{1}$ and Rodica-Mariana Ion $^{3}$ \\ ${ }^{1}$ Faculty of Technical Physics, Poznań University of Technology, Nieszawska 13A, 60-965 Poznań, Poland \\ ${ }^{2}$ Department of Biology and Enviromental Studies, K. Marcinkowski University of Medical Sciences, \\ Długa 1/2, 61-848 Poznań, Poland \\ ${ }^{3}$ ZECASIN Splaiul Independentei 202, 79611 Bucharest, Romania
}

\begin{abstract}
The time-resolved photothermal methods are used in order to establish the yield of intersystem (singlet-triplet) crossing of dyes that are candidates for the application in photodynamic therapy. It is known that molecules with long living triplet state cause efficient photosensitization by direct interaction with the cells or by singlet oxygen generation.

The steady state photoacoustic spectra enables one to establish the dye incorporation into cells.

Both methods were applied to several phthalocyanines and the results gathered by photothermal measurements were compared with the results obtained by means of other methods.The six investigated dyes were compared.
\end{abstract}

\section{INTRODUCTION}

Phthalocyanines (Pcs) are promising candidates to the application in photodynamic therapy (PDT) because they exhibit the absorption band in a region of $650 \mathrm{~nm}-$ $800 \mathrm{~nm}$ in which tissue is rather transparent $[1,2]$.

The destruction of neoplastic (malignant) cells, in both mechanisms: type 1-direct interaction of sensitizer with tissue and type 2-interaction with created singlet oxygen $\left({ }^{1} \mathrm{O}_{2}\right)$, is done through the triplet state of sensitized dye (Figure 1) [1, 2].

Therefore efficient sensitizer should have possibly high yield of triplet state formation.

The yield of triplet states generation by intersystem crossing from excited singlet state (Figure 1) can be evaluated by the measurements of the slow component of thermal deactivation (TD) of excitation using laser induced optoacoustic spectroscopy (LIOAS) [3, 4]. The sensitizer molecules must to be more efficiently incorporated into malignant than in healthy cells. It is possible to apply several spectral methods in order to establish the efficiency of dye incorporation into tissue. As we will demonstrate the steady state photoacoustic spectroscopy is very convenient way to check the amount of dye molecules incorporated into cells [5]. The incorporation of dye into stimulated and resting human peripherical blood mononuclear cells (PBMC) were compared. The stimulated cells are the model of malignant cells. The spectral properties of sensitizers in incubation solvent and in rigid matrix have been also investigated $[2,4,5]$.

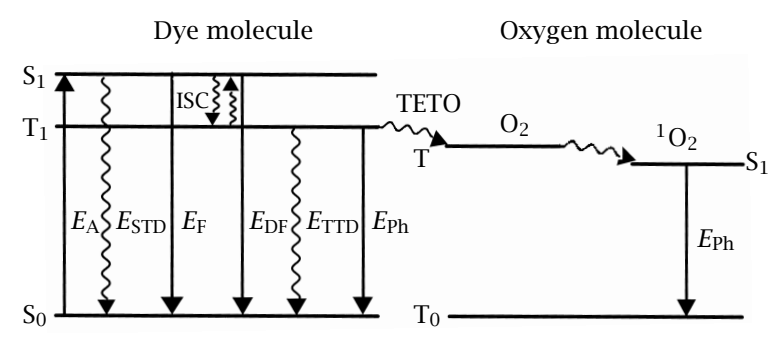

Figure 1. Jabłoński diagram. $\mathrm{S}_{0}$ - ground singlet state, $\mathrm{S}_{1}$ excited singlet state, $\mathrm{T}_{1}$ - triplet state, $E_{A}$ - absorption energy, $E_{\mathrm{F}}$ - fluorescence energy, $E_{\mathrm{DF}}$ delayed fluorescence energy, $E_{\mathrm{STD}}$ and $E_{\mathrm{TTD}}$ - singlet and triplet thermal deactivation, $E_{\mathrm{Ph}}$ - phosphorecence energy, TETO - triplet energy transfer to oxygen, $\mathrm{O}_{2}$ - oxygen in triplet state, wave-shape lines-non radiative transitions.

\section{LASER INDUCED OPTOACOUSTIC SPECTROSCOPY}

Figure 2 shows the scheme of the arrangement used for the LIOAS measurements. Sample is illuminated by the flash from dye-nitrogen laser. Laser light energy is measured by pyrroelectric energy radiometer. The sample is located in the temperature-controlled cuvette-holder. The piezoelectric transducer with $1 \mathrm{MHz}$ frequency resolution is attached to the cuvette wall. The waveform thermal signal (Figure 3) is memorized using digital oscilloscope. Such wave-form LIOAS signals for the measured sample as well as for the reference dye were measured. An example of such results is shown in Figure 3. 


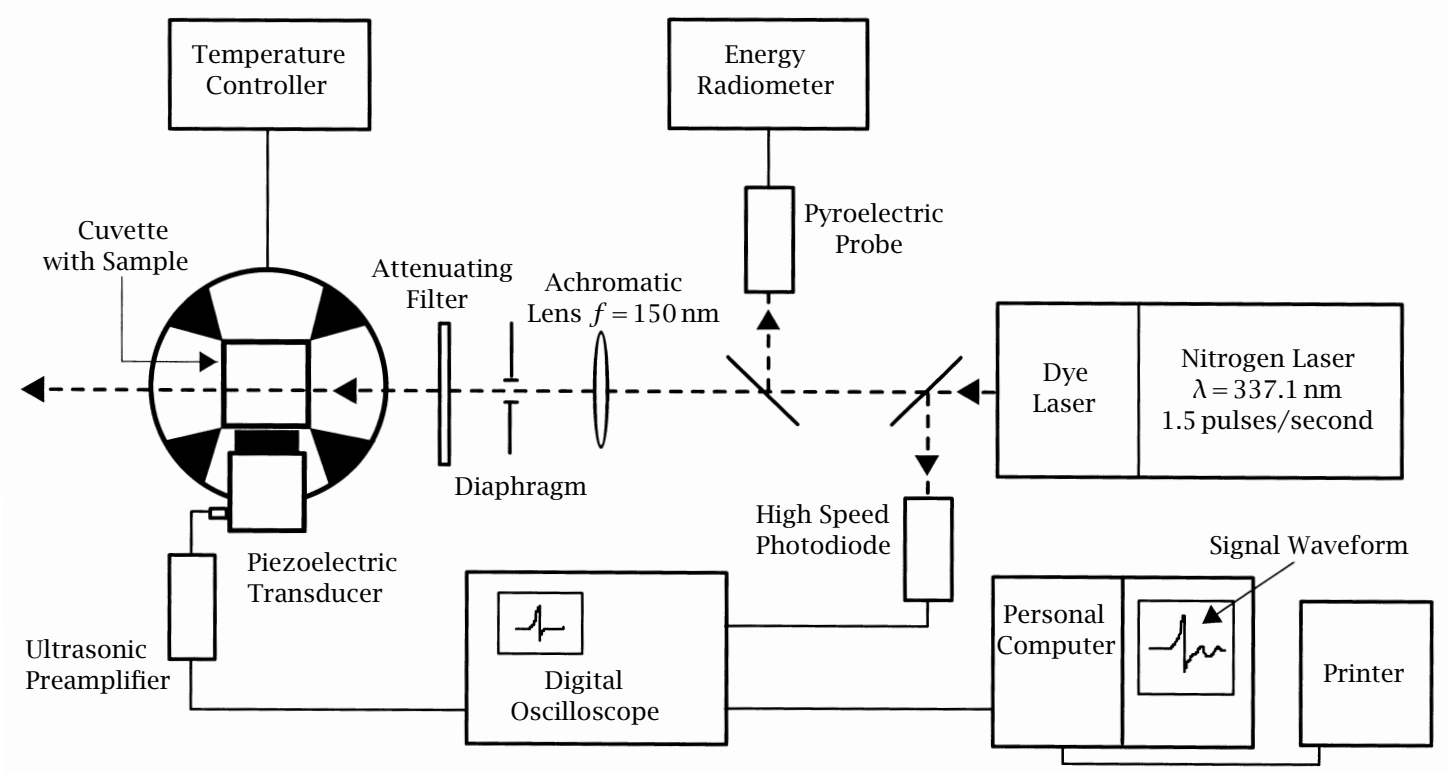

Figure 2. Arrangement for the measurement of time resolved photothermal effects.

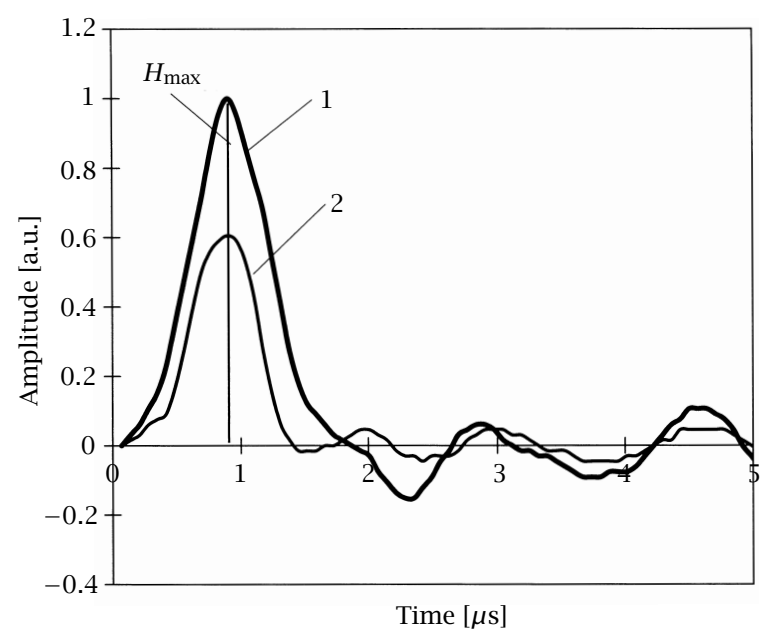

Figure 3. Waveform LIOAS signal for reference sample (curve 1) and analyzed samples (ZnPc in DMSO ) (curve 2).

The reference dye was chosen on the basis of the literature [3]. It deactivates all excitation energy in time shorter than the time resolution of used arrangement (about $0.6 \mu \mathrm{s}$ ) [3, 5]. It is possible to suppose that this deactivation occurs promptly.

The wave-form LIOAS signals were analyzed using approximated method proposed by Marti et al. [6], as well as deconvoluted by means of program elaborated by Rudzki-Small and Small [7].

In the first method the amplitude of first maximum of wave-form signal ( $H_{\max }$ in Figure 3 ), obtained at the same amount of absorbed quanta for measured sample and for reference, are compared. Such procedure gives opportunity to evaluate the part of the energy that is deactivated thermally promptly and the part that is deactivated slowly (it means in time longer than time resolution of the apparatus but quicker, in our arrangement, than time about $5 \mu \mathrm{s}$ ). Such slow deactivated energy is generated predominantly by thermal deactivation of triplet states populated by intersystem crossing from the excited singlet state. The amplitude of first maximum $H_{\max }$ is:

$$
H_{\max }=k \alpha E_{\text {las }}\left(1-10^{-A}\right),
$$

where $\alpha$ is part of energy promptly deactivated into heat, $E_{\text {las }}$ is energy of laser light, $A$ is the absorption of sample at wavelength of laser pulse, $k$ is the coefficient related to apparatus optical geometry, electronic impendence and thermoelastic properties of sample. The $k$-value is the same for the sample and reference, $\alpha$ for the reference is approximated to be equal unity. Therefore from the measurements done for the sample and for the reference, the value of $\alpha$ for the sample can be obtained. In order to calculate the yield of triplet state generation $\left(\phi_{\mathrm{T}}\right)$ we can use the formula:

$$
\phi_{\mathrm{T}} E_{\mathrm{T}}=(1-\alpha) E_{\mathrm{las}}-\phi_{\mathrm{F}} E_{\mathrm{F}},
$$

where the $\phi_{\mathrm{F}}$ is the yield of dye fluorescence, $E_{\mathrm{F}}$ the energy of fluorescence and $E_{\mathrm{T}}$ the energy of triplet state. $E_{\mathrm{F}}$ and $E_{\mathrm{T}}$ are obtained from literature or are experimentally established.

The application of both, Marti et al. [6] and RudzkiSmall and Small [7], methods provides additional information concerning investigated systems. 
(a)

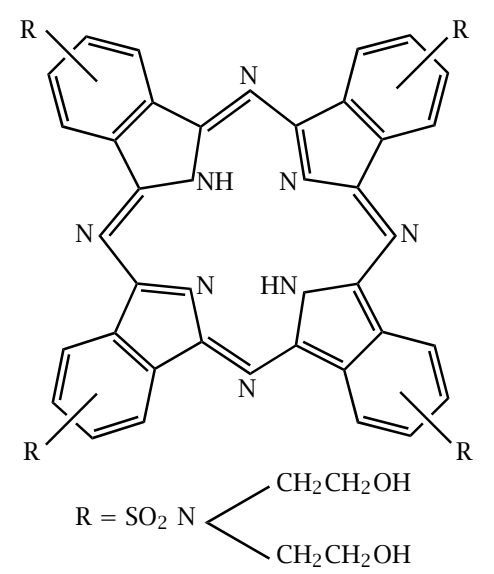

(c)

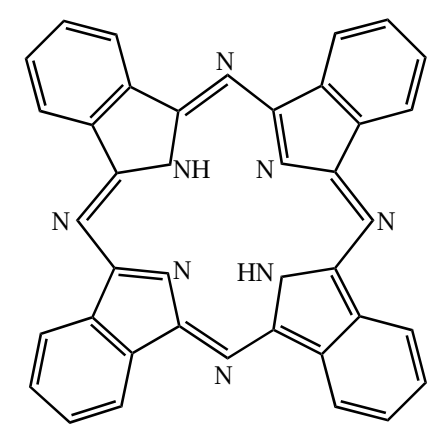

(e)

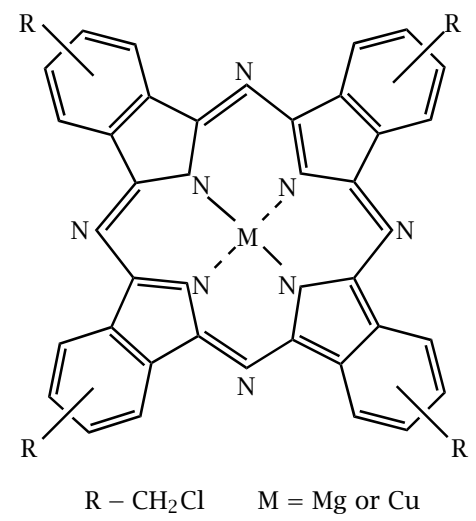

(b)

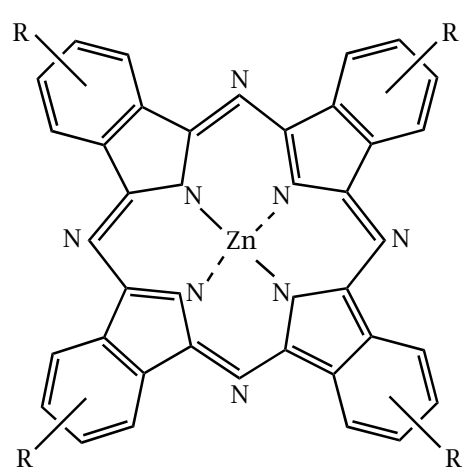

(d)

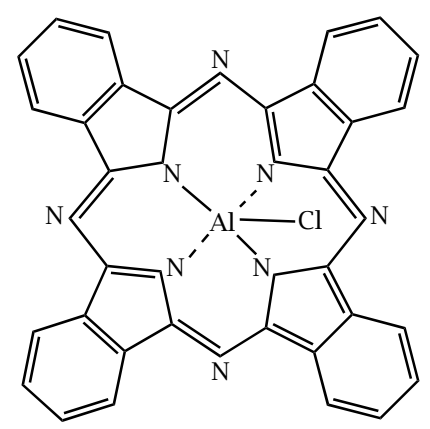

(f)<smiles>C/C=c1/c2cccc(Sc3ccccc3)c2/c(=C/C2=C/C(=N\C)c3c(Sc4ccccc4)cccc32)n1[N+](C)(C)Cl</smiles>

Scheme 1. The structures of investigated Pcs: (a) Solar Pc, (b) ZnPc, (c) $\mathrm{H}_{2} \mathrm{Pc}$, (d) AlPcCl I, (e) MgPc or CuPc, (f) AlPcCl II.

\section{STEADY STATE PHOTOACOUSTIC SPECTROSCOPY}

The photoacoustic spectra (PAS) were taken using a single beam spectrometer [2, 5, 8].

The sample placed in a photoacoustic cell was illuminated by modulated light. The heat generated in a sample reached the surface between sample and working gas. The thin layer of warmed gas works as a piston on the rest of the gas and generates the photoacoustic wave acting on a microphone. The acoustic signal was preamplified and sent to phase lock-in amplifier. The reference signal for the amplifier is provided by a chopper. The signal is computer processed. To eliminate the influence of the spectral distribution of the used lamp the sample spectrum is divided by the spectrum of carbon black.

\section{RESULTS}

Scheme 1 presents the structure of investigated Pcs. They had been investigated incorporated into the 

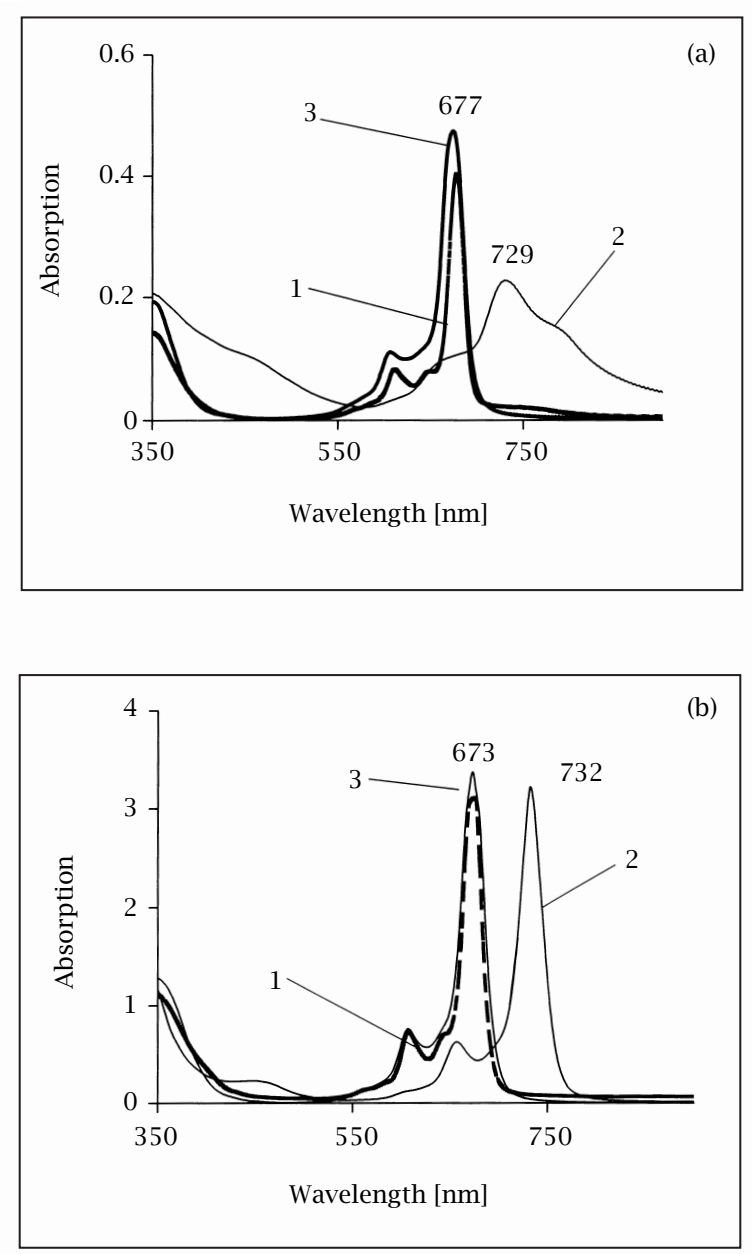

Figure 4. Absorption spectra of some Pcs: (a) in PVA, (b) in DMSO. Curves: 1-ZnPc, 2-AlPcCl II, 3-Solar Pc.

cells $[4,5]$ using the dimethyl sulfoxide (DMSO) as incubation solvent. The Pcs properties were also investigated in polyvinyl alcohol (PVA) films (the absorption spectra of ZnPc, AlPc Cl II and Solar Pc are shown in Figure 4(a)). To PVA film Pcs were introduced from DMSO solution. Figure 4(b) shows the absorption of the same Pcs in DMSO. From the comparison of the Figure 4(a) with Figure 4(b) it follows that the embedding the dyes into rigid matrix can change the shape of the absorption spectra of some Pcs. Similar effect may occur as a result of dyes incorporation into the cells.

On the ground of LIOAS measurements the yields of the generation of triplet states $\left(\phi_{\mathrm{T}}\right)$ for the investigated dyes increases according the following sequence: Solar Pc $<$ CuPc $<\mathrm{H}_{2}$ Pc $<$ AlPcCl II $<$ MgPc $<$ AlPcCl I $<$ ZnPc. The lowest $\phi_{\mathrm{T}}$ equals 0.35 and is observed for Solar Pc, whereas the highest value (about one) is obtained for ZnPc. It means that in the case of embedding of the same amount of given dye molecules in the cells the most effective photodestruction would be done by ZnPc and only a weak effect will be due to the illumination of cells stained by Solar Pc. To be sure that slow TD component is due to triplet states thermal deactivation the lifetime of this process is established by deconvolution of wave form curves of sample and reference. The deconvolution results for $\mathrm{ZnPc}$ has shown that about $30 \%$ of absorbed light energy is deactivated in longer time than time resolution of the arrangement, with an average decay time $(\tau)$ about $7 \mu \mathrm{s}$. This percent is increasing in a solution bubbled by nitrogen in which oxygen concentration is much lower. The difference in values of yields of slow component of thermal deactivation obtained by two methods of analysis (almost 100\% in first method and 30\% in a second) is due to different approximations: in Marti et al. [6] method all the slow processes are obtained by the elimination of quick processes described by first maximum $H_{\max }$ (Figure 3), whereas in Rudzki-Small and Small deconvolution [7] only the amplitude of the slow component at given decay time is counted. All processes slower than $5 \mu$ s are not included because of limitation of experimental method [3].

High efficiency of triplet state generation is not the only condition which has to be fulfilled by good sensitizer, it has also to be efficiently incorporated into malignant cell, less efficiently to healthy cells, not to be toxic and also possibly quickly has to be expended from organisms. The efficiency of the dye incorporation can be among other spectral methods established on the ground of PAS of stained cells.

Figure 5 shows the examples of steady state photoacoustic spectra of Pcs. Steady state PAS (Figure 5) shows the PAS of ZnPc and MgPc in DMSO measured at the same frequency of light modulation and the incident light intensity are different.

The ZnPc PAS signal is much higher than that of MgPc. The yield of thermal deactivation (TD), calculated as the ratio of PAS signal to absorbed light energy, is for ZnPc also higher than that for MgPc. It means that before using the PAS maxima for investigation of dye content in the cells we have to establish TD of investigated dyes. The TD yield depends on the dye interaction with surrounding. In the case of the tissue, when the dyes are located in similar parts of cell, the ratio of their TD yields should be similar as in other media. The measurements by the PAS of the content of the dyes in the cells are more convenient than the application of the absorption spectra, because PAS results are less perturbed by light scattering than absorption [2, 10]. Therefore the presence of dye molecules can be easier established on the ground of PAS than on the basis of absorption spectra.

The fluorescence yields of Pcs in the cells are about one order lower than that in incubation solvent [5] therefore in some cases it is not easy to establish 


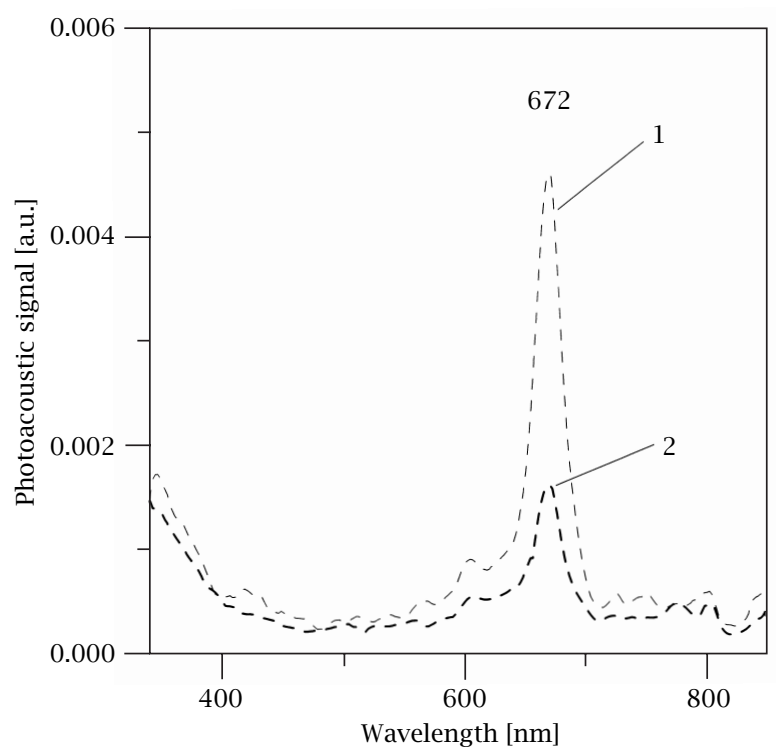

Figure 5. Steady state of photoacoustic spectra of Pcs in DMSO: curve 1 - ZnPc, curve 2 - MgPc. Frequency of light modulation $15 \mathrm{~Hz}$.

the amount of incorporated dye on the basis of fluorescence spectra [5]. Fluorescence and TD are competing processes therefore the dyes with high value of fluorescence yield (such as e.g. MgPc) exhibit low values of TD (Figure 5). In case of efficiently fluorescent molecules the application of the emission spectra can be more convenient than measurements of PAS. Therefore the measurements of fluorescence of stained cells can be also useful for the investigation of dye incorporation. Figure 6 presents fluorescence spectra of the PBMC stained by ZnPc [10]. Emission is excited in two regions: at $405 \mathrm{~nm}$ where absorption of inner cell material is higher than that of the dye and at $610 \mathrm{~nm}$ or $680 \mathrm{~nm}$ where dye absorption predominants. The fluorescence measurements can be much more sensitive method for the establishment of dye incorporation than the absorption especially when auto fluorescence of cell material and dye emission are located in different spectral regions. Similarly as in the case of PAS measurements the yields of dyes fluorescence in the incubation solvent have to be also established.

It follows from cell fluorescence (Figure 6) stained by ZnPc that this dye is incorporated more efficiently into activated than into resting cells. From this point of view ZnPc seems to be suitable for photodynamic therapy. There is a large difference in the emission in a region $400 \mathrm{~nm}-550 \mathrm{~nm}$ (due predominantly to the fluorescence of the cell material) between stained resting cells (curve 1) and activated cells (with dye-curve 2, and without dye curve 3 ). This is expected result because the activation should perturb the cell structure. Figure 7 shows the fluorescence spectra of PBMC stained by

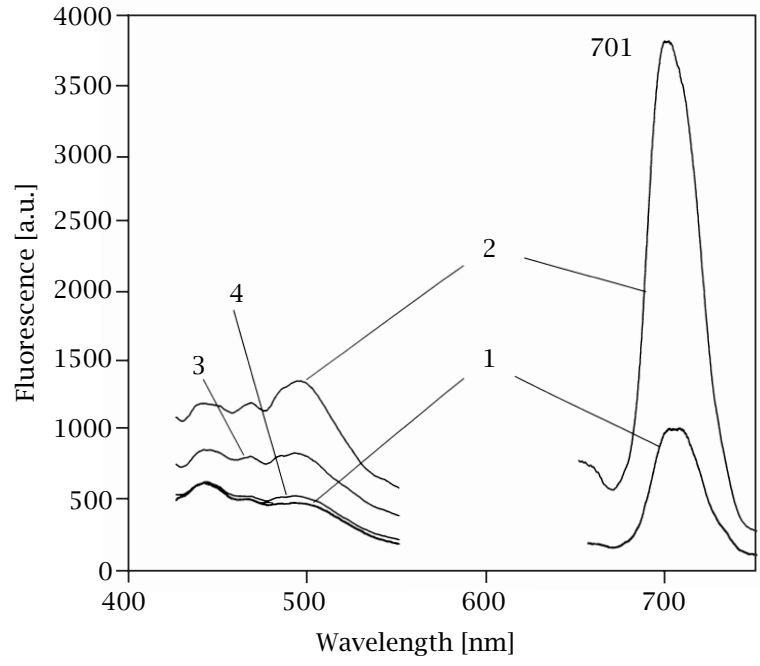

Figure 6. Fluorescence spectra of PBMC. Spectra in a region 400-500 $\mathrm{nm}$ were obtained by $405 \mathrm{~nm}$ excitation; spectra in a region $650-750 \mathrm{~nm}$ at $610 \mathrm{~nm}$ excitation. Curve: 1 - resting cells stained by ZnPc; 2 - stained by ZnPc activated cells; 3 - cells in DMSO with activator only; 4 - cells in DMSO only.

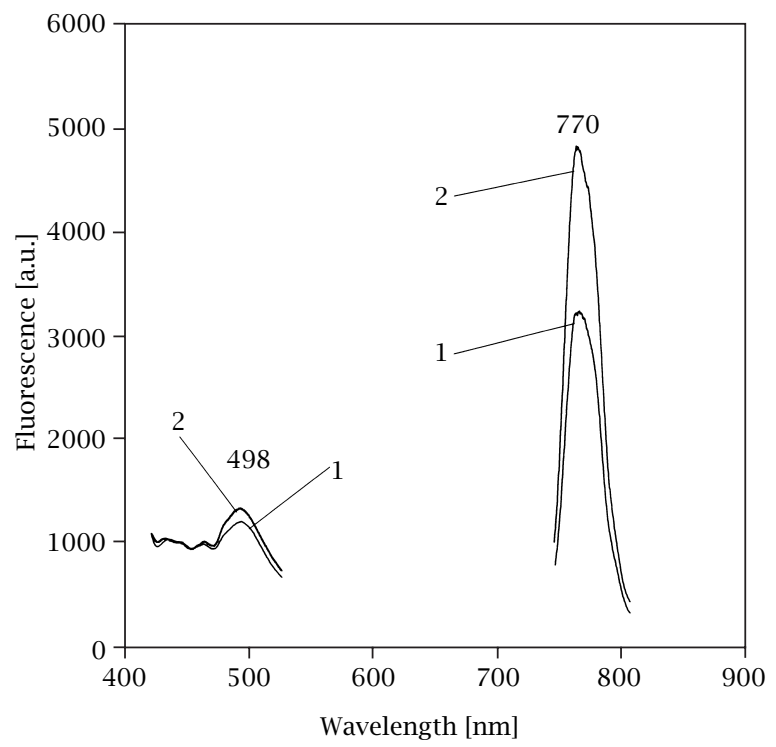

Figure 7. Fluorescence spectra of cells stained by AlPcClII, 1resting cells, 2-activated cells. Emission in a region: $750 \mathrm{~nm}-$ $800 \mathrm{~nm}$-excited at $680 \mathrm{~nm}$; in a region $420 \mathrm{~nm}-520 \mathrm{~nm}-$ excited at $405 \mathrm{~nm}$.

AlPcCl I. This dye exhibits lower selectivity than ZnPc (Figure 6) in introduction both for resting and activated cells.

The selection of optimal Pcs need further investigations, but it was shown that the combination of absorption, fluorescence and photothermal methods can be helpful in such investigations. 


\section{CONCLUSIONS}

It follows from our investigations that the selectivity of incorporation of ZnPc into activated and resting cells is very high. This dye exhibit also very efficient generation of triplet states. The metal free Pcs have low efficiency of triplet generation therefore even in a case of incorporation into activated cells may be less efficient in photodynamic action.

From our measurements it follows that photothermal methods can be very useful when we have to establish which dye can to be best candidate for the application in photodynamic therapy. The LIOAS gives the opportunity to help us evaluate the yield of triplet state generation, whereas the steady state PAS can be used for evaluation of the efficiency of the dye incorporation into cells.

\section{ACKNOWLEDGEMENTS}

The paper was supported by the state Committee for Scientific Research (KBN) grant No 4PO5B 10617.

\section{References}

[1] I. Rosenthal, Photochem. Photobiol. 53 (1991), 859.
[2] D. Frąckowiak, A. Planner, and K. Wiktorowicz, Near-infrared applications in medicine, in NearInfrared Applications in Biotechnology, R. Raghavachari (ed.), Marcel Dekker, Inc. New York, Basel, 2001, pp. 151-183.

[3] S. E. Braslavsky and G. E. Heibel, Chem. Rev. 92 (1992), 1381.

[4] D. Frąckowiak, A. Planner, A. Waszkowiak, A. Boguta, R. M. Ion, and K. Wiktorowicz, J. Photochem. Photobiol. A: Chemistry 141 (2001), 101.

[5] D. Frąckowiak, A. Waszkowiak, H. Manikowski, R. M. Ion, J. Cofta, and K. Wiktorowicz, Acta Biochim. Polon. 48 (2001), 257.

[6] C. Marti, S. Nonell, M. Nicolaus, and T. Torres, Photochem. Photobiol. 71 (2000), 53.

[7] J. Rudzki-Small, L. J. Libertini, and E. W. Small, Biophys. Chem. 42 (1992), 29.

[8] D. Ducharm, A. Tessier, and R. M. Leblanc, Rev. Sci. Inst. 5 (1979), 1461.

[9] D. Frąckowiak, M. Niedbalska, and K. Wiktorowicz, J. Photochem. Photobiol. B: Biology 33 (1996), 45.

[10] A. Waszkowiak, D. Frąckowiak, K. Wiktorowicz, and J. Miyake, Biophysical Chemistry (2001), submitted. 


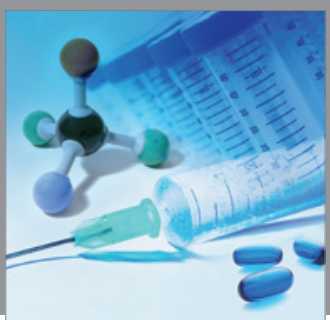

International Journal of

Medicinal Chemistry

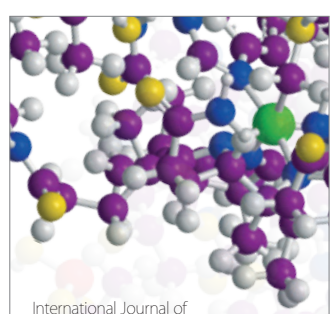

Carbohydrate Chemistry

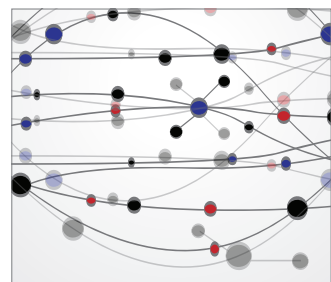

The Scientific World Journal
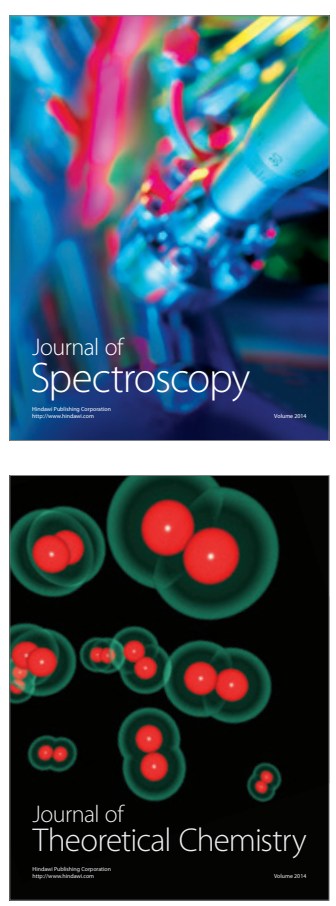
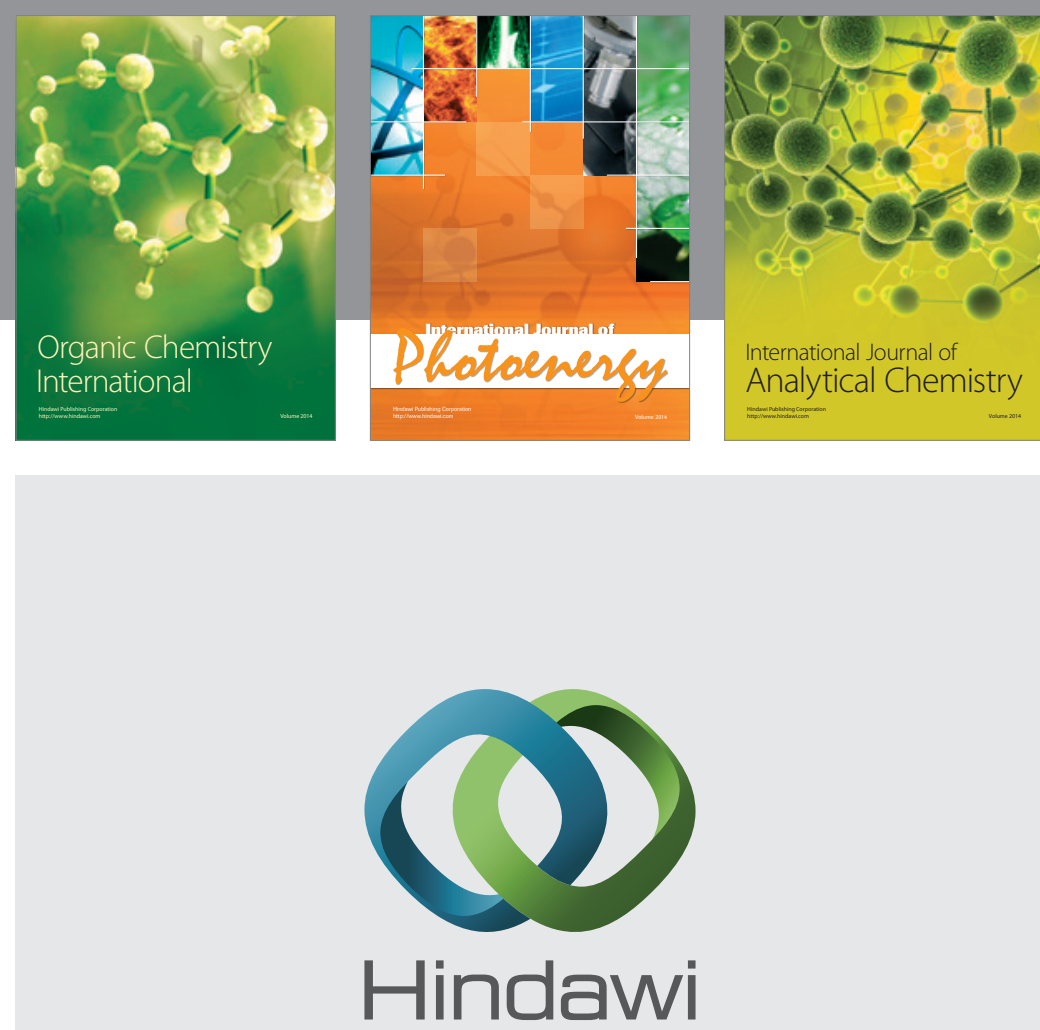

Submit your manuscripts at

http://www.hindawi.com
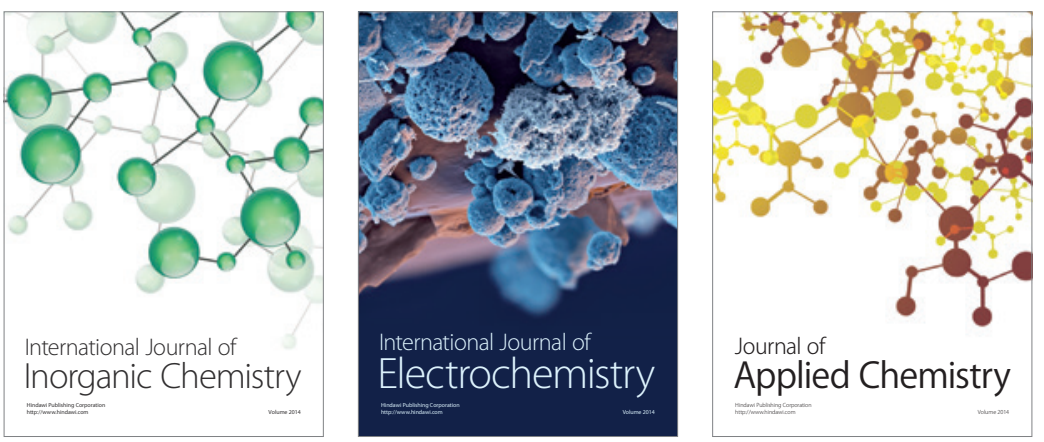

Journal of

Applied Chemistry
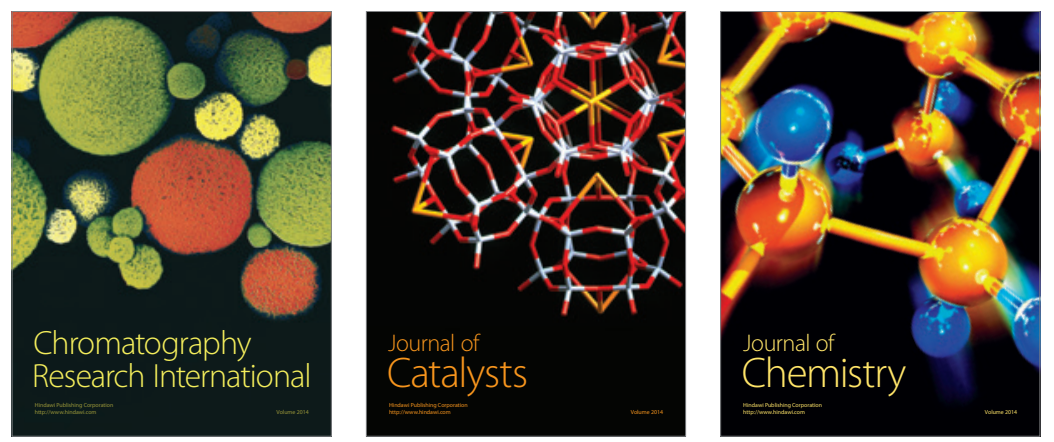
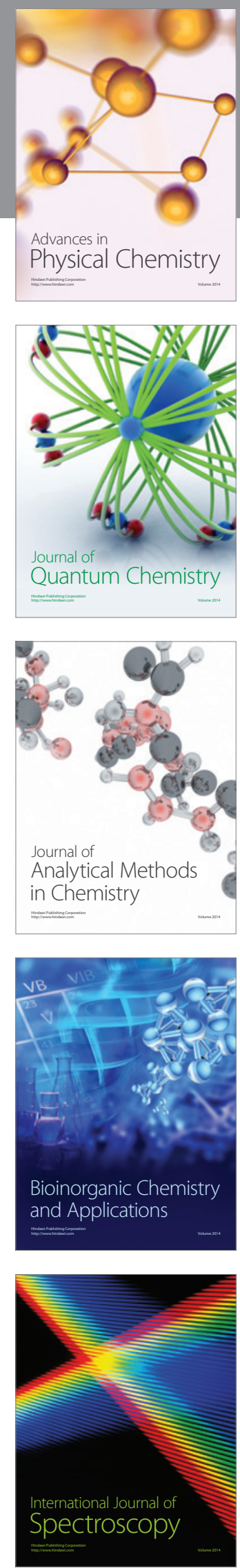